\title{
A CRITICAL TEST FOR THE PURITY OF GASES
}

\author{
By Martin Shepherd
}

\section{ABSTRACT}

This paper reports a method by which the purity of gases (and liquids having normal boiling points below room temperatures) may be critically tested. The method involves one approximately isothermal distillation, whereby a small initial distillate, a middle cut, and a small final residue are obtained. The pressures of these three fractions are intercompared by means of a simple differential manometer and form the basis for indicating impurities of higher and lower boiling points than that of the approximately pure substance.

The method eliminates the exacting temperature requirements of the widely used criterion of constancy of pressure during an isothermal condensation or vaporization. At the same time, it offers more information than the isothermal condensation, and is suitable for testing mixtures containing more than two components. In many cases the order of magnitude of the impurities may be calculated. Like the isothermal condensation, it is not suitable for studying azeotropic mixtures.

The preparation of a pure gas is not an easy matter, and satisfactory tests for purity are often difficult to formulate and usually difficult to perform. In many cases the method of preparation is about as good a guarantee of purity as the method of test subsequently applied, and sometimes it offers even more reliable assurance of purity. These complications are largely responsible for the fact that reported investigations deal abundantly with gases of doubtful purity.

The ordinary methods of so-called "exact" volumetric gas analysis, which are often employed in the effort to establish the purity of a gas, are subject to errors of such magnitude as to be unfit for this purpose. It is sometimes possible to design very special chemical methods which may offer satisfactory evidence of purity. In general, however, the measurement of one or more of the physical properties of a gas affords the basis for the determination of its purity.

One of the most frequently employed criteria for purity is the constancy of pressure during isothermal condensation or vaporization. The method is very useful, but has limitations which are not generally recognized. Washburn ${ }^{1}$ has discussed the physical limitations of this method.

In this connection, Washburn suggested a more sensitive and convenient method for determining purity in case the substance to be examined is nearly pure. This suggestion is given on page 595 (paragraph 3) of his paper, and forms the basis of the method described in the present discussion. The apparatus and detailed technique, which have been found suitable for the application of this method, are reported in order to make the method easily available. At the same time, the original suggestion has been extended in order to permit the collection of additional information.

$1 \mathrm{E} . \mathrm{W}$. Washburn, Constancy of pressure during isothermal condensation or vaporization as a criterion of purity, Zs. physik. Chemie (Cohen-Festband), p. 592, 1927. 
One of the exacting demands of the method of isothermal condensation is the required maintenance of very constant temperatures over relatively long periods. An elaborate cryostat is consequently necessary. It is a relatively simple matter to avoid this difficult requirement, and at the same time to improve greatly the sensitivity of the test for purity. Furthermore, it is possible to extend the information obtained by establishing the presence or absence of impurities having boiling points both higher and lower than that of the approximately pure substance. The identity of these impurities can sometimes be established, and if the mixture is limited to three components, the order of magnitude of the amounts present may be determined in many cases.

The method is as follows. One simple approximately isothermal distillation is conducted. The vapor pressure of a very small portion

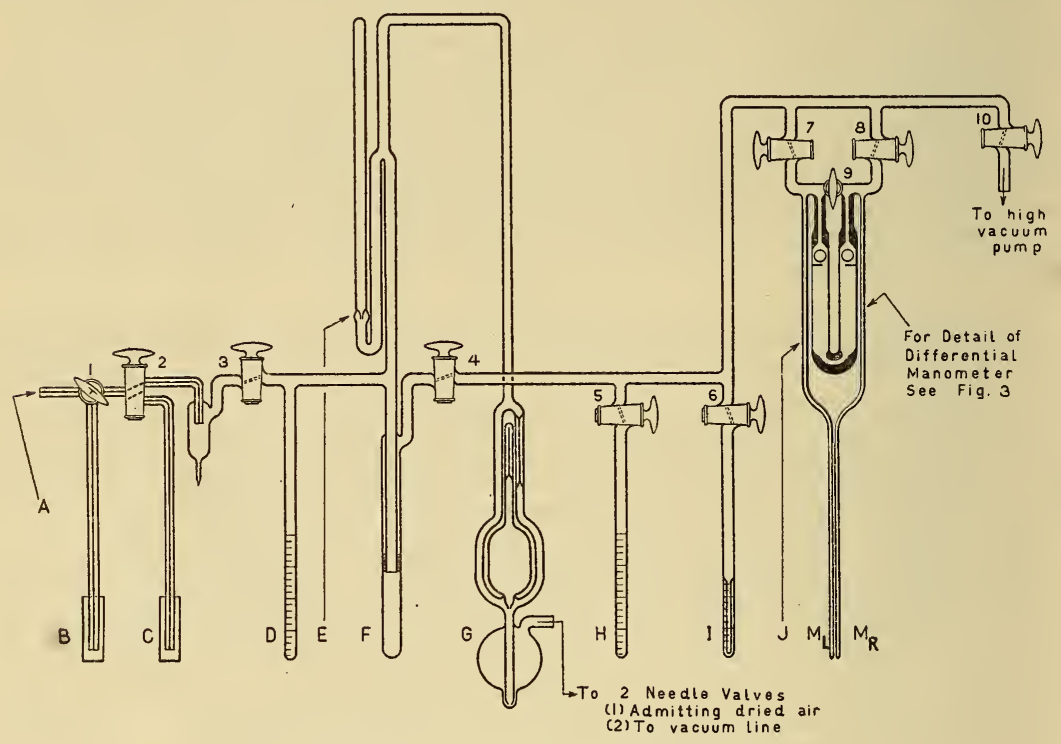

FIGURE 1.-Assembly of apparatus.

of the initial distillate is compared differentially with that of a small portion of the middle cut, and the vapor pressure of the latter fraction is in turn compared differentially with that of the small final residue. The method retains one of the limitations of the isothermal condensation, in that it is not suitable for azeotropic mixtures. Another limitation is that the approximately pure substance condense (at pressures not greatly exceeding atmospheric) at temperatures ordinarily available. The practical lower limit in most laboratories would be about $-210 \mathrm{C}$ (liquid air boiling under reduced pressure).

The apparatus required is not extensive and can be constructed more easily than a good cryostat. The assembled unit is illustrated in figure 1 .

The procedure is best followed by referring to this diagram. The whole apparatus is thoroughly evacuated until no pressure increase is indicated by the McLeod gage, G, after the mercury vapor pump has been shut off at cock 10 for several hours. 
The gas sample to be tested is introduced at A. The arrangement of stopcocks 1 and 2 and mercury seals B and C permits necessary variations in the method of transferring the sample. The sampling connections may be flushed with gas or mercury through C. The seal B acts as a manometer to insure positive pressure (if desired) during the transfer, and also provides a safety outlet if the sample is stored under pressure. If the sample container is sealed to A, the connections may be evacuated. The amount of sample and method of storing will determine the desirable procedure.

The sample is condensed in the graduated tube D. The amount to be condensed will depend upon the volume of gas available for the test, and, if a sufficient amount is at hand, two or three $\mathrm{ml}$ of condensate may be taken. The volume of the condensate is noted at the condensing temperature. The same temperature should be used subsequently for condensing the small fractions in the differential manometer. Liquid air is often convenient for this purpose, although solid carbon dioxide or other suitable cooling agents are sometimes required.

The condensate is next transferred (by vaporizing and condensing) to the distilling tube $F$. Its pressure (measured by the barometric manometer $E$ ) at liquid-air temperature may sometimes indicate a nitrogen or air impurity in a substance whose vapor pressure is very low at this temperature. The distilling tube is now surrounded by a snugly fitting copper block (fig. 2) adjusted to the proper temperature for the approximately isothermal distillation. This metal envelop is suspended in a dewar tube and cooled to the desired temperature. It has sufficient heat capacity to maintain this temperature with little attention. The distillation should be conducted at low pressures (the order of a few $\mathrm{mm}$ ). A thorough discussion
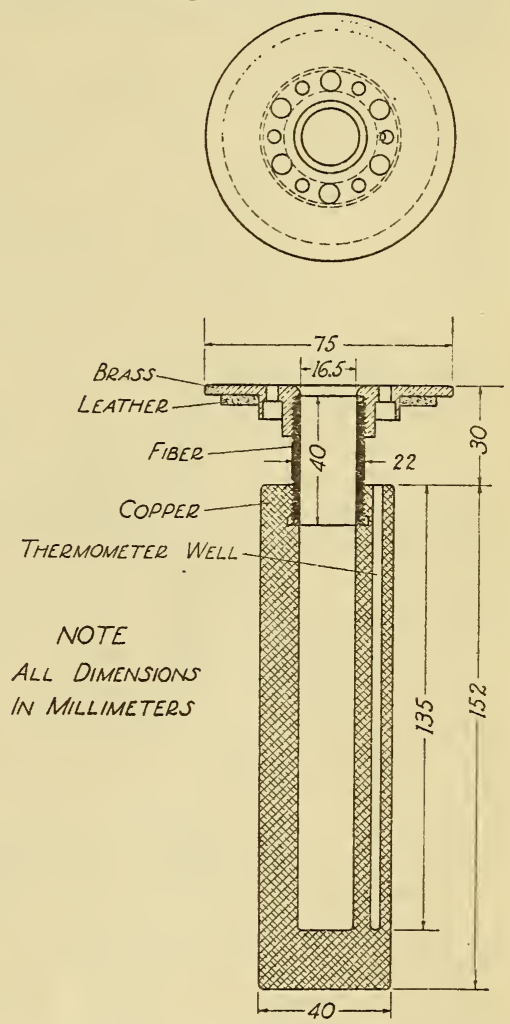

Figure 2.-Detail of metal envelop for distilling tube.

of the procedures employed for this type of distillation is given in a previous report. $^{2}$

The initial distillate is condensed in the graduated tube $I$ at the temperature previously used to condense the entire sample (a temperature much lower than that of the distilling tube). Its volume is noted and compared with that of the total sample. The optimum volume of initial distillate (or final residue) is the smallest amount which can be transferred to the differential manometer with assurance

${ }_{2}^{2}$ Martin Shepherd, B.S. Jour. Research, vol. 2 (RP75), P. 1145, 1929. 
that it will not be completely vaporized at the temperature employed for the measurement of vapor pressure differences. Greater sensitivity is thus obtained. This optimum may be determined from the approximate values for the capacity of the manometer above the condensate, the density of the liquid at the condensing temperature and the vapor pressure at the temperature used. When the proper amount of initial distillate has condensed in $I, 4$ is closed and 8 opened to transfer the distillate to the right-hand condensing bulb $\left(M_{R}\right)$ attached to the differential manometer $J . \quad M_{R}$ is then isolated by closing 8. The vapor pressure of the initial distillate (within the range of the manometer) may now be measured. This measurement is optional and is desirable only in case it may indicate the nature of the lower boiling impurity.

Approximately one half of the condensate in $F$ is now distilled and condensed in $H$. Cock 4 is closed and this fraction is pumped off and discarded, leaving $H$ and $I$ evacuated. The distillation is resumed until a small portion of the middle cut has been collected by condensation in $I$. It is then transferred to the left-hand condensing bulb $\left(M_{L}\right)$ of the manometer, which is isolated by closing 7 . The difference (if any) in vapor pressure between the initial distillate and middle cut is measured at a temperature near the normal boiling point of the approximately pure substance. The procedures for using the differential manometer will be discussed later.

The initial distillate is now discarded to the pump, leaving $M_{R}, H$, and $I$ evacuated. At this point the vapor pressure of the middle cut may be measured. The distillation is again resumed until the major portion of the residue in $F$ has been collected in $H$. The last small final residue is condensed in $I$. If its volume happens to be too large, it may be immediately transferred to $F$, another portion distilled to $H$ and the smaller residue again collected in $I$. From $I$ it is transferred to $M_{R}$, where its vapor pressure is compared with that of the middle cut by reading the differential manometer. If desired, the middle cut may be pumped off and the vapor pressure of the final residue measured. If the purity of the substance is very high, the vapor pressures of the initial distillate and final residue should be compared directly for the highest sensitivity.

A secondary check on the differential manometer may be made by observing the vapor pressures of the condensate in $F$, before and after distilling off the initial distillate and middle cut, and before removing the final residue. It is, of course, necessary to adjust the distilling temperature to the same value for these observations, and this cannot always be done precisely nor can proper equilibrium always be assured.

The differential manometer is shown in figure 3 . Its construction is very simple. The positions of the mercury menisci are read by two hollow micrometer heads or gages (not shown in fig. 1) which are cemented onto the arms of the manometer. The rotating cylinders of these gages are engraved with vernier scales exactly as in an ordinary micrometer gage. The readings are taken in the same manner as with the better class of barometers. The manometer can be read to $0.01 \mathrm{~mm}$. Since both arms are read, the limiting precision is about $0.02 \mathrm{~mm}$, although the actual accuracy obtained will depend largely upon errors caused by differential capillarity. A $10 \mathrm{~mm}$ bore will meet the average requirement, although larger diameters are sometimes necessary. Results with oxygen, for example, indicated the inade- 
quacy of the $10 \mathrm{~mm}$ size. If a systematic capillarity error is suspected, the relative positions of the condensates may be interchanged by transferring from $M_{R}$ to $I, M_{L}$ to $M_{R}$, and $I$ to $M_{L}$.

The mercury is prevented from large accidental displacements by two check valves. These are simply spherical glass balls ground to seat against a conical grinding in the tube above.

The zero reading of the manometer is taken (before transferring any distillate) with both sides evacuated. Stopcock 9 or 7 and 8 should be opened. A check is made after adjusting the temperature around the condensing bulbs of the manometer. At the end of a determination, cock 9 may be opened to check the zero reading. (The use of cock 9 is optional. It is not shown in fig. 3.)

The entire accuracy of the differential vapor pressure measurement depends upon securing a very uniform temperature around the condensing bulbs of the manometer. The temperature need not be accurately measured, accurately adjusted to any fixed value, nor even be constant. But it must be the same around and in each condensing bulb. For this reason, the bulbs terminate at the same level and touch one another. Even so, it is often impossible to secure uniform temperature around them when they are immersed in a liquid bath even though it may be vigorously stirred. This difficulty was eliminated by surrounding the bulbs with a tube containing mercury, which freezes as a solid block around them at low temperatures. When

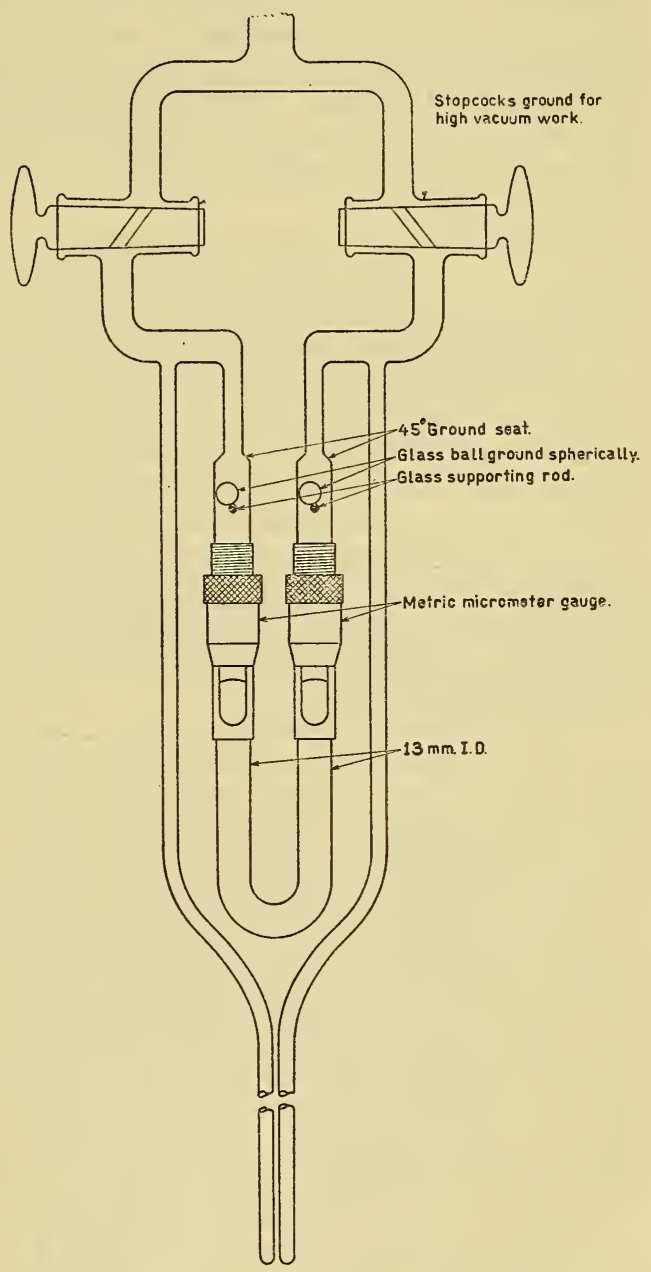

FIgURE 3.-Detail of differential manometer. this is done, the pressures remain quite steady and no fluctuations are observed. The mercury can be cooled as desired by liquid air which is added to the dewar tube surrounding $M_{L}$ and $M_{R}$. This insulation prevents any sudden temperature change outside of the frozen mercury.

The precautions to be observed in using the differential manometer are simple. Enough condensate must be taken so that some liquid will be present at the temperature employed for the differential vapor pressure measurement. If too much condensate is taken, the surface 
of the liquid may be above that of the surrounding mercury and the observed pressure will be too high. The error may be large in either of the two cases mentioned. If the prescribed technic is followed, the meniscii of the two condensates will be at the same level.

Another precaution which must sometimes be taken is to insure the removal of nitrogen or air from the middle cut and final residue. It is sometimes desirable to remove these impurities from the initial distillate, if their presence is satisfactorily established and the possible presence of another impurity of lower boiling point than that of the approximately pure substance is sought. For example, in testing a sample of propane, it is desirable to remove nitrogen in order to be able to detect ethane. Nitrogen is usually removed with the portion discarded before taking the middle cut, but in case of doubt, the small condensates in $M_{R}$ and $M_{L}$ may be held at liquid-air temperature while pumping.

Finally, if the temperature selected for the comparison of vapor pressures is too low, the manometer cannot be read with the required sensitivity. This will become apparent in the following discussion.

The information obtained may be listed:

(1) The pressure of the initial condensate at liquid air temperatures, which in some cases may indicate the presence of nitrogen or air. These two impurities are frequently present. Other gases of low boiling points, may, of course, be indicated, but the likelihood of their presence is often known from the history of the sample.

(2) The difference between the vapor pressure of a small initial distillate and the vapor pressure of the middle cut, measured at a temperature near the normal boiling point of the approximately pure substance. This at once establishes the possible presence of lower boiling impurities.

(3) The difference between the vapor pressure of the middle cut and the vapor pressure of a small final residue, measured at a temperature near the normal boiling point of the approximately pure substance. This will establish the possible presence of higher boiling impurities.

(4) The vapor pressure of the initial distillate, which may sometimes indicate the nature of the lower boiling impurity.

(5) The vapor pressure of the final residue, which may sometimes indicate the nature of the higher boiling impurity.

If the higher and lower boiling impurities are each single substances and their vapor pressures are known at some temperature near the normal boiling point of the pure substance, and if these impurities may be assumed to obey Raoult's law, the approximate amounts of each can be calculated. For this purpose, it will also be necessary to assume that the isothermal distillation achieved a sharp separation of the higher and lower boiling impurities, which will not always be true although the middle cut will often be free from lower and higher boiling impurities. If sharp separation was not attained, the calculation proposed will not indicate the maximum amount of impurity, but simply the difference in purity of the two fractions involved, from which it is possible to state there is at least a certain amount of the impurity present.

The expression by means of which the difference in purity between any two fractions may be calculated is derived from Raoult's law. Let $A$ and $B$ identify the substances of the two-component systems composing the fractions, $p^{o}{ }_{A}$ and $p^{o}{ }_{B}$ their respective vapor pressures 
in the pure state, and $X_{A}$ and $X_{B}$ their mole fractions. Then, if $p$ is the total vapor pressure of the mixture,

$$
p=p^{o}{ }_{A} X_{A}+p^{o}{ }_{B} X_{B}=p^{o}{ }_{A}\left(1-X_{B}\right)+p_{B}^{o} X_{B} \text {. (Raoult) }
$$

Such an equation may be written for each fraction, the subscripts 1 and 2 identifying the two fractions:

$$
\begin{aligned}
& p_{1}=p^{o}{ }_{A}\left(1-X_{B_{1}}\right)+p^{o}{ }_{B} X_{B_{1}}, \\
& p_{2}=p^{o}{ }_{A}\left(1-X_{B_{2}}\right)+p^{o}{ }_{B} X_{B_{2}} .
\end{aligned}
$$

The difference in the vapor pressures of the two fractions obtained by subtracting equation (3) from equation (2) is

$$
p_{1}-p_{2}=-p^{o}{ }_{A} X_{B_{1}}+p^{o}{ }_{B} X_{B_{1}}+p^{o}{ }_{A} X_{B_{2}}-p^{o}{ }_{B} X_{B_{2}},
$$

which reduces to

$$
p_{1}-p_{2}=\left(X_{B_{1}}-X_{B_{2}}\right)\left(p_{B}^{O}-p_{A}^{o}\right)
$$

The quantity $p_{1}-p_{2}$ is the differential vapor pressure measured by the manometer, and $X_{B_{1}}-X_{B_{2}}$ is the difference in purity which was sought. For convenience these terms may be called $\Delta p$ and $\Delta X_{B}$ respectively. Equation (5) then becomes

$$
\Delta X_{B}=\frac{\Delta p}{p^{O}{ }_{B}-p^{O}{ }_{A}}
$$

Washburn has derived an equation (unpublished report) which has a more general application since it takes account of Henry's law as well as that of Raoult. The form of the equation is the same as the one given above, but $K_{B}$, the Henry's-law constant for the impurity $B$, is substituted for $p_{B}{ }_{B}$ :

$$
\Delta X_{B}=\frac{\Delta p}{K_{B}-p^{0}{ }_{A}}
$$

The difference in purity between two fractions is given by equation (6). If $X_{B}$ in the middle cut may be assumed to be zero (which is frequently true), $\Delta X_{B}$ of the equation gives the amount of the impurity measured in the initial distillate (or in the final residue). If most of the impurity $B$ is contained in the initial distillate (or final residue), $\Delta X_{B}$ will indicate an amount approaching that of the actual impurity. If the above conditions do not obtain, and any $\Delta p$ is measured, $\Delta X_{B}$ gives an amount of impurity less than the actual amount present. Since, in addition to the above, the ratio of the volume of the initial distillate (or the final residue) to the total sample is known, it is sometimes possible to compute the amount of impurity present in the original sample.

It is apparent that the difference $p^{o}{ }_{B}-p^{o}{ }_{A}$ must be reasonably large if $\Delta X_{B}$ is small, and $\Delta p$ is to be easily measurable. Thus if it is desired to detect 0.0001 mole fraction of impurity, $p^{O}{ }_{B}-p^{o}{ }_{A}$ should be at least $500 \mathrm{~mm}$ (preferably more) in order that $\Delta p$ may be measurable. For this reason, the temperature at which $\Delta p$ is measured is chosen accordingly. It is obvious that the method becomes insensitive without modification of the manometer to determine impurities whose normal boiling points are too close to that of the approximately pure substance.

Washington, November 20, 1933. 